\title{
NEUROPATÍA VASCULÍTICA NO SISTÉMICA: REVISIÓN DE CASO
}

OSCAR CASTRO ANGULO ${ }^{1}$, HERNÁN CASTRO VARGAS², LUISA FERNANDA GUZMÁN MOLANO³

\author{
${ }^{1}$ Residente de Neurología IV año. Hospital Militar Central/ Universidad Militar Nueva Granada. \\ ${ }^{2}$ Neurólogo. Fellowship Neurofisiología. Universidad del Rosario Escuela de Medicina y Ciencias de la Salud. \\ ${ }^{3}$ Especialista Servicio de Neurología. Hospital Militar Central/ Universidad Militar Nueva Granada.
}

Correspondencia: Hernán Castro Vargas. Transversal 3 \# 49-00 piso 3 sur, Bogotá, Colombia. hernan.castrov@urosario.edu.co. Teléfono (57) 3114004920

Recibido: 27 de octubre de 2017 Aceptado: 5 de diciembre de 2017

\section{Resumen}

La neuropatía vasculítica no sistémica es una condición poco común, caracterizada por el compromiso aislado del sistema nervioso periférico a causa de la infiltración celular en el lecho vascular encargado de su irrigación. Es un término acuñado hace poco más de tres décadas y se considera una enfermedad poco descrita y subdiagnósticada.

Se presenta el caso clínico de un paciente masculino de mediana edad que debuta con síntomas progresivos de predominio motor en miembros inferiores, quien es sometido a una serie de estudios que permiten concluir el diagnóstico. Es tratado con corticoides sistémicos, agentes citotóxicos y, por último, agentes biológicos con los que se estabilizaron los síntomas.

Palabras clave: vasculitis; neuropatía; electrodiagnóstico; inmunosupresor; biopsia de nervio; remisión; resistencia.

\section{NON-SYSTEMIC VASCULITIC NEUROPATHY: CASE REVIEW}

\section{Summary}

Non-systemic vasculitic neuropathy is a rare condition characterized by the isolated involvement of the peripheral nervous system caused by cellular infiltration in the vascular bed responsible for its irrigation. It is a term which was coined a little over three decades ago and is considered a poorly described and underdiagnosed disease. Below, we present the clinical case of a middle-aged male patient who began experiencing progressive symptoms of motor predominance in the lower limbs, and who underwent a series of studies in order to conclude a diagnosis. The patient was treated with systemic corticosteroids, cytotoxic agents and, finally, biological agents which stabilized the symptoms.

Keywords: vasculitis; neuropathy; electrodiagnosis; immunosuppressant; nerve biopsy; remission; resistance. 


\section{NEUROPATIA VASCULÍTICA NÃO SISTÊMICA: REVISÃO DE CASO}

\section{Resumo}

A neuropatia vasculítica não sistêmica é uma condição pouco comum, caracterizada pelo compromisso isolado do sistema nervoso periférico a causa da infiltração celular no leito vascular encarregado de sua irrigação. É um termo cunhado há pouco mais de três décadas e se considera uma doença pouco descrita e subdiagnosticada. A seguir, se apresenta o caso clínico de um paciente masculino de meia idade que debuta com sintomas progressivos de predomínio motor em membros inferiores, que é submetido a uma série de estudos que permitem concluir o diagnóstico. É tratado com corticoides sistêmicos, agentes citotóxicos e, por último, agentes biológicos com o que se estabilizaram os sintomas.

Palavras-chave: vasculite; neuropatia; eletro diagnóstico; imunossupressor; biopsia de nervo; remissão; resistência.

\section{Introducción}

La neuropatía vasculítica no sistémica o aislada es una condición poco frecuente, cuyo diagnóstico exige haber descartado condiciones vasculares primarias o secundarias como causa dicha condición. El compromiso se limita al sistema nervioso periférico. El estudio de electrodiagnóstico y biopsia de nervio constituyen ayudas diagnósticas indispensables. El inicio de tratamiento inmunosupresor inmediato es indispensable para disminuir el déficit neurológico consecuente, la lista de medicamentos disponibles incluye los corticoides sistémicos, algunos agentes citotóxicos, agentes biológicos como la IGIV y rituximab, sin embargo, la recuperación completa es poco frecuente.

\section{Caso clínico}

Paciente masculino de 41 años, que en el año 2009 inicia cuadro clínico de pérdida progresiva de la fuerza de miembro inferior izquierdo asociada a disminución de la masa muscular, que genera limitación para la marcha y bipedestación por paraparesia. Logra bipedestación con caminador, se moviliza en silla de ruedas, presenta deterioro lento y progresivo de la fuerza muscular.

En 2010 , se realiza estudio de electromiografía y velocidades de neuroconducción que reporta ausencia de potencial motor en nervio peroneo izquierdo y disminución de la amplitud motora en nervio tibial izquierdo con disminución de la velocidad de conducción. La electromiografía de rectus femoris, tibial anterior y paravertebral
L5 izquierdo muestra la presencia de PUM con duración y amplitud aumentadas, presencia de actividad denervatoria caracterizada por la presencia de ondas agudas y fasciculaciones en el reposo. El estudio neurofisiológico presenta resultados anormales, lo que sugiere enfermedad motoneuronal y lleva a considerar compromiso por mononeuropatía múltiple. Un año después el mismo estudio no reporta progresión del cuadro motoneuronal, aunque se evidencia mayor compromiso en miembro inferior izquierdo.

En 2013, se lleva a cabo el estudio de biopsia de nervio periférico con hallazgos histológicos sugestivos de arteriopatía de pequeño vaso severa, asociada a estudio de ACE (enzima convertidora de angiotensina) negativo.

En 2014, es hospitalizado para estudio de causas sistémicas de vasculitis. En el examen neurológico se encuentra disminución del tono muscular e hipotrofia en miembros inferiores, de predominio izquierdo. La fuerza muscular en miembros inferiores se registra como $3+/ 5$ miembro inferior derecho, 3/5 miembro inferior izquierdo, ROT $+/++++$ rotulianos, $0 /++++$ aquilianos, sensibilidad tacto superficial y vibratoria conservadas, respuesta plantar flexora bilateral; no se reportan otras alteraciones en el examen físico.

Los estudios de química sanguínea reportan: PT 10,9; INR 0,96; PTT 28; TSH 5,3; leucocitos 9,1; N 59\%; L 32\%; Hb 17; PLT 264; VDRL no reactivo; vitamina B12 418; glucemia 79; BUN 12; K 3,8 y HbA1C 5,4. No se identifican alteraciones en estudios imagenológi- 
cos de tórax, abdomen y neuroeje. No presenta evidencia de alteraciones en estudio de punción lumbar.

Durante la hospitalización se inicia manejo con IGIV en un ciclo de cinco días con adecuada tolerancia, posteriormente es llevado a cinco hospitalizaciones durante los siguientes meses para aplicación de nuevos ciclos de IGIV. Durante este periodo no se registra deterioro de la fuerza muscular de miembros inferiores ni nuevas manifestaciones. En el año 2016 se inicia manejo con rituximab 1000 mg IV semestral.

\section{Neuropatía vasculítica}

\section{Definición}

Las vasculitis son desórdenes sistémicos o locales en los cuales las paredes de los vasos sanguíneos son infiltradas por células inflamatorias, generando lesiones isquémicas secundarias en el tejido irrigado. Los vasos afectados pueden ser de cualquier calibre (1); el sistema nervioso periférico se puede ver comprometido en $60-70 \%$ de los casos de vasculitis sistémicas dando lugar a las neuropatías vasculíticas $(2,3)$.

La clasificación más aceptada para este grupo de patologías es la propuesta por Peripheral Nerve Society Task Force que considera tres grupos basándose en el origen de la neuropatía vasculítica $(2,3)$ :

1. Primaria sistémica: el daño vascular es producido por condiciones inherentes a los vasos sanguíneos como en el síndrome de Churg Strauss y la granulomatosis de Wegener (1-3).

2. Secundaria sistémica: el daño vascular es mediado por el sistema inmune, puede ser desencadenado por infecciones, medicamentos, neoplasias, condiciones preexistentes autoinmunes como las enfermedades del tejido conectivo (1-3).

3. No sistémica: el daño vascular no se asocia a condiciones primarias o secundarias sistémicas, el compromiso se limita al sistema nervioso periférico $(1,3,4)$. Es también denominada neuropatía vasculítica aislada (5). Este tipo de neuropatía vasculítica fue descrita por primera vez en 1985, sin embargo, aún es pobremente entendida y probablemente subdiagnósticada si se compara con las variantes sistémicas. El término fue acuñado por BJ Dyck en 1987, algunos alegan que es una forma leve de vasculitis sistémica con síntomas de predominio nervioso periférico, mientras que otros argumentan que es una enfermedad únicamente del nervio periférico (6).

\section{Fisiopatología}

Los vasos sanguíneos que irrigan el sistema nervioso periférico son infiltrados por células inflamatorias, causando isquemia endoneural y daño axonal; los vasos del epineuro se ven comprometidos con mayor frecuencia (4). Los nervios periféricos tienen una rica irrigación anastomótica con dos sistemas vasculares integrados que, asociada a la habilidad del nervio para tolerar condiciones anaeróbicas, favorece la resistencia a situaciones de isquemia crónica, por eso solo cuando el compromiso de la vasa nervorum es extenso se presentan daños axonales (3). Las fibras mielinizadas son las más sensibles a isquemia, por ende, su compromiso se manifiesta más temprano (5).

Las fibras motoras y sensitivas se pueden afectar en un patrón asimétrico, el daño puede perdurar a pesar de la inactividad de la vasculitis, la recuperación suele ser lenta y parcial, haciendo que el tratamiento temprano sea necesario para prevenir el daño permanente $(1,5)$.

\section{Manifestaciones clínicas}

Las manifestaciones clínicas de las neuropatías vasculíticas dependen de la distribución y severidad del compromiso vascular. En casos primarios y secundarios los síntomas sistémicos, como el dolor abdominal, hematuria y síntomas respiratorios, son comunes. Los casos no sistémicos no se acompañan de otros síntomas, aunque $30 \%$ de los pacientes reporta manifestaciones constitucionales como fatiga, pérdida de peso, mialgias, artralgias o fiebre (1-3).

Los sintomas se pueden desarrollar en un periodo de semanas a meses, en el $30 \%$ de los casos la instauración es lenta y progresiva. Estos pacientes suelen debutar con ataques agudos de dolor, seguidos de sensación de ardor cutáneo y debilidad local que comprometen el territorio de un solo nervio; en un lapso de tiempo que oscila entre días y semanas otros nervios se pueden ver afectados lo que resulta en mononeuritis múltiple, algunos pacientes pueden presentar varias mononeuropatías que se combinan en un patrón de polineuropatía simétrico o asimétrico. La mayoría de los pacientes presentan síntomas sensitivos y motores, $80 \%$ de los casos debutan con dolor, en $15 \%$ de las personas afectadas predominan los síntomas sensitivos por el compromiso de nervios cutáneos, la presentación motora pura es rara $(1-3,6,7)$. 
El fenotipo típico de la neuropatía vasculítica no sistémica descrito en $45 \%$ de los casos es el de una neuropatía multifocal o mononeuropatía múltiple por eventos isquémicos secuenciales en nervios individuales, $35 \%$ de los pacientes presenta mononeuropatías individuales que se pueden superponer semejando una polineuropatía asimétrica, solo $20 \%$ asemeja una polineuropatía simétrica. El nervio peroneo común o la división peronea del nervio ciático distal en miembros inferiores y el nervio ulnar próximo al codo en miembros superiores tienen pobre circulación colateral, por lo tanto, son más susceptibles a sufrir daño por isquemia $(1-4,6)$.

\section{Diagnóstico}

Una correcta anamnesis, asociada a un examen físico completo, constituye una herramienta valiosa en el diagnóstico de las neuropatías vasculíticas, las ayudas diagnósticas disponibles son las siguientes (8):

1. Laboratorio clínico: estudios paraclínicos que arrojan información de importancia relacionada con estados inflamatorios, infecciosos, autoinmunes y neoplásicos que pueden asociarse al origen de la vasculitis (8); los recomendados son el hemograma, electrolitos, función renal, uroanálisis, VSG, PCR, ANA, ANCA, factor reumatoide, electroforesis de proteínas, PTOG, HbA1C, crioglobulinas, complemento, antígeno de superficie de hepatitis $\mathrm{B}$ y anticuerpos hepatitis $\mathrm{C}$. Se sugiere considerar otros estudios de química sanguínea individualizados a cada caso. El estudio de punción lumbar está indicado en pacientes con signos proximales y síntomas que sugieran compromiso radicular, evidencia electrodiagnóstica de enfermedad desmielinizante o axonal, sospecha de sarcoidosis, infección o neoplasia (8).

2. Electrodiagnóstico: el estudio de electromiografía y neuroconducción ayuda a definir la distribución del compromiso neurológico (focal, multifocal), identifica la modalidad (sensitiva o motora) y permite caracterizar la probable patología (desmielinizante o pérdida axonal), además de ayudar a seleccionar el nervio idóneo para estudio de biopsia. En las neuropatías vasculares se encuentran hallazgos sugestivos de pérdida axonal aguda y subaguda en fibras sensitivas y motoras, frecuentemente en parches o distribución multifocal. El patrón más frecuente es el de polineuropatía asimétrica, seguido de patrón de mononeuropatía múltiple y, en menor frecuencia, el patrón de mononeuropatía de pérdida axonal (2). Puede encontrarse un bloqueo en estudios de conducción nerviosa motora en los estadios siguientes al inicio de los síntomas, lo que sucede porque la degeneración walle- riana no se ha desarrollado en la parte distal del nervio infartado, por lo que permite la conducción de potenciales de acción a través del nervio en la estimulación distal, en contraste con la falla en conducción a través del nervio infartado con estimulación proximal. Cuando sucede la degeneración walleriana después de una semana, la falla en la conducción ocurre en la estimulación proximal y distal y desaparece ese aparente bloqueo. Los potenciales de acción sensitivos disminuyen en amplitud y desaparecen entre siete y diez días después del evento isquémico (2). En los casos más crónicos puede haber evidencia de denervación en curso o varios grados de reinervación (4).

3. Biopsia de nervio: la biopsia de nervio continúa siendo crucial en el diagnóstico de neuropatías periféricas. Es usada de manera amplia en el diagnóstico de neuropatías de origen vasculítico, inflamatorio, neoplásico o por depósitos. En caso de neuropatías de fibras pequeñas la biopsia de piel es el estudio ideal (8), se sugiere asociarla a biopsia muscular para mayor precisión diagnóstica $(4,7,8)$. Los hallazgos más frecuentes son inflamación de la pared del vaso y signos de daño vascular activo o crónico, la inflamación perivascular o mural no es específica $(2,3)$. En los casos de neuropatía vasculítica no sistémica la biopsia de nervio suele mostrar cambios consistentes en neuropatía axonal que incluyen disminución en la densidad de la fibra nerviosa, degeneración walleriana y regeneración en clústeres axonales; la pérdida axonal suele ser centro fascicular en áreas proximales, pero suele ser multifocal en áreas distales por el entretejido de las fibras descendentes; suele afectar pequeños vasos, pero no se limita a la microvasculatura $(3,9)$. Las lesiones vasculíticas se caracterizan por infiltrados de células $\mathrm{T}$ (CD4 o CD8) en el epineuro y macrófagos que invaden las paredes de los vasos sanguíneos y producen necrosis fibrinoide y otros signos de daño vascular; la inmunofluorescencia directa muestra depósitos inmunes de IgM, fibrinógeno y C3 $(3,4,9)$.

\section{Criterios diagnósticos para neuropatía vasculítica no sistémica según el Peripheral Nerve Task Force} 1. Neuropatía vasculítica definida por patología o probablemente por clínica.

2. Evaluar signos síntomas y paraclínicos que sugieran compromiso sistémico, en caso de estar presentes se descarta: evidencia de compromiso sistémico, aneurismas viscerales, ANCAS, VSG $>100$, crioglobulinas, evi- 
dencia patológica de vasculitis en tejido no neuromuscular, infección subyacente, condiciones o medicamentos que predisponen el desarrollo de vasculitis $(3,9)$

\section{Tratamiento}

Los casos de vasculitis sistémica asociada a un desencadenante específico requieren tratamiento para la condición o desencadenante identificado (3), el inicio temprano de la terapia disminuye el daño inflamatorio (2). Es de importancia destacar que las neuropatías vasculíticas se relacionan con pérdida axonal, a pesar de un manejo inmunomodulador agresivo, la reinervación y recuperación sensitiva y motora pueden ser lentas e incompletas, la meta a corto plazo es estabilizar y a largo plazo se busca disminuir el déficit neurológico teniendo en cuenta que la recuperación completa es poco frecuente (9).

Los casos sistémicos que suelen ser de mayor severidad requieren la combinación de corticoides y agentes citotóxicos como ciclofosfamida, azatioprina, metotrexato. En casos no sistémicos severos o que sean progresivos a pesar del uso óptimo de corticoides se recomienda asociar también un agente citotóxico $(3,9)$.

Se recomienda tener siempre en cuenta las medidas no farmacológicas como consejería y educación, dispositivos de soporte y manejo del dolor neuropático (3).

El tratamiento en general tiene dos fases:

La inducción de la remisión es el tratamiento inicial que resulta en la resolución de las manifestaciones de vasculitis activa (7).

El mantenimiento de la remisión es el tratamiento continuo por tiempo prolongado (18-24 meses) con el objetivo de mantener el control y prevenir recaídas (7).

Las dosis sugeridas de prednisona o prednisolona es $1 /$ $\mathrm{mg} / \mathrm{kg} /$ día. En casos severos se sugiere la administración metilprednisolona por 3-5 días seguido de prednisolona oral, después de 1-2 meses se comienza a disminuir la dosis en 5-10 mg cada semana según la respuesta del paciente (2). Los casos de neuropatía vasculítica no sistémica son susceptibles de recibir monoterapia con corticoides en una dosis de prednisolona $1 \mathrm{mg} / \mathrm{kg} /$ día y titular a $25 \mathrm{mg}$ a los 3 meses, $10 \mathrm{mg}$ a los 6 meses y continuar bajas dosis (prednisona 5-7,5 mg/día) por 6-18 meses para prevenir recaídas $(2,3,9)$.
Los medicamentos citotóxicos recomendados son:

Ciclofosfamida a una dosis de $2 \mathrm{mg} / \mathrm{kg} /$ día oral o 15 $\mathrm{mg} / \mathrm{kg}$ IV cada 2-3 semanas, se reemplaza por azatioprina o metotrexato una vez alcance el estado de remisión, generalmente en 3-6 meses (2). Se deben tener en cuenta los posibles efectos secundarios: supresión de médula ósea, cistitis hemorrágica, infecciones sistémicas, riesgo de malignidad como linfoma, leucemia carcinoma de células transicionales de la vejiga y cáncer de piel no melanoma, por lo que se debe hacer hemograma cada semana tras la primera dosis y después cada dos semanas, parcial de orina cada semana por los primeros meses y después mensual $(2,10)$.

Azatioprina en dosis de $50 \mathrm{mg} /$ día y aumentar $50 \mathrm{mg}$ cada 3-5 días hasta $2-3 \mathrm{mg} / \mathrm{kg} /$ día. Los efectos secundarios esperados son náuseas, diarrea, mialgias, rash y fiebre al inicio del tratamiento, a largo plazo puede generar supresión de médula ósea, hepatotoxicidad, pancreatitis y riesgo de infecciones sistémicas y neoplasia, por lo que debe monitorizar con hemograma y estudio de función hepática cada semana por el primer mes, después cada mes por 6 meses y después cada 3 meses $(2,10)$.

Metotrexato puede ser útil en casos leves. Se recomienda iniciar con 7,5-15 mg/semana y aumentar hasta 20$25 \mathrm{mg} / \mathrm{semana}$ según la respuesta clínica, se debe continuar por 18-24 meses antes de intentar disminuir su dosis. Puede generar nauseas, leucopenia, estomatitis ulcerativa, fatiga, mareo, rash, supresión de médula ósea, neumonitis intersticial, por lo anterior se debe monitorizar enzimas hepáticas, no olvidar suplementar con ácido fólico en una dosis no inferior a $5 \mathrm{mg}$ a la semana $(2,10)$.

La resistencia al tratamiento se define como el aumento en la actividad de la enfermedad después de 6 semanas de terapia combinada, o mejoría, pero con persistencia de actividad de la enfermedad después de 8 semanas (3), en estos casos se recomienda confirmar el diagnóstico o dar paso a terapias biológicas como la IGIV o rituximab $(2,3)$.

La IGIV ha mostrado ser un tratamiento efectivo en varios pacientes con resistencia a terapia estándar, la dosis sugerida es de $2 \mathrm{~g} / \mathrm{kg}$ en $2-5$ días, de acuerdo con la respuesta se da las siguientes dosis; suele ser bien tolerado, pero puede generar meningitis aséptica, cefalea, mialgias, lesión renal aguda, neutropenia, rash o síndrome de hiperviscosidad (2). 
Rituximab es una inmunoglobulina monoclonal anti CD20, la dosis recomendada es $375 \mathrm{mg} / \mathrm{m} 2$ IV cada semana por 4 semanas, puede generar hipotensión, disnea, fiebre, náuseas, escalofrío, prurito, urticaria, angioedema, cefalea o enfermedad pulmonar (2).

\section{Seguimiento}

En los casos de vasculitis sistémicas, según la causa, existen parámetros inflamatorios o serológicos que permiten hacer seguimiento a la actividad de la enfermedad. En los casos no sistémicos estas medidas no están disponibles, sin embargo, para ambas clasificaciones la mejoría del dolor, la fatiga y astenia y los cambios reportados en el examen neurológico son de utilidad para el correcto seguimiento a la evolución ya que permite detectar la estabilidad del cuadro o posibles recaídas $(3,4,11)$.

\section{Conclusión}

La neuropatía vasculítica no sistémica es una condición infrecuente cuyo abordaje implica el uso de la semiología como una herramienta diagnóstica inicial y valiosa, asociada a ayudas diagnósticas disponibles que permiten evidenciar el daño del lecho vascular en el sistema nervioso periférico, asociado a la ausencia de compromiso sistémico. El diagnóstico y tratamiento oportuno son imprescindibles para estabilizar los síntomas y disminuir el déficit neurológico. Conocer el grupo de agentes farmacológicos disponibles, sus contraindicaciones $y$ posibles efectos secundarios es fundamental para el abordaje de los pacientes, teniendo en cuenta que la recuperación completa es poco frecuente.

\section{Conflicto de intereses}

Los autores del presente trabajo declaran no tener ningún tipo de conflicto de intereses que invalide la investigación.

\section{Apoyo financiero}

Este trabajo se realizó con recursos propios de los investigadores.

\section{Referencias}

1. Vrancken, ALlexander; Said G. Vasculitic neuropathy. In: Handbook of Clinical Neurology. 2013. p. 463-83.

2. Gwathmey KG, Burns TM, Collins MP, Dyck PJB. Vasculitic neuropathies. Lancet Neurol [Internet]. 2014; 13(1): 67-82.

3. Collins MP, Arnold WD, Kissel JT. The neuropathies of vasculitis. Neurol Clin. 2013; 31(2): 557-95.

4. Ubogu EE, Immunopathology N. Inflammatory Neuropathies: Pathology, molecular markers and targets for specific therapeutic intervention. Acta Neuropthologica. 2016; 130(4): 445-68.

5. Rutgers A, Kallenberg CGM. Peripheral neuropathy in AAV - when vasculitis hits a nerve. Nat Publ Gr [Internet]. 2011; 8(3): 127-8.

6. Collins MP, Periquet MI, Heumatology EXR. Isolated vasculitis of the peripheral nervous system. Clin Exp Rheumatol. 2008; 26(49): S118-s30.

7. Marsh EA, Davies LM, Llewelyn JG. How to recognise and treat peripheral nervous system vasculitis. Pract Neurol. 2013; 13: 408-11.

8. Grisold W, Grisold A. Neuromuscular Issues in Systemic Disease. Curr Neurol Neurosci Rep. 2015; 15(7): 2-12.

9. Collins MP. The vasculitic neuropathies: an update. Curr Opin Neurol. 2012; 25: 573-85.

10. Broussalis E, Trinka E, Mccoy M, Killer M. Treatment strategies for vasculitis that affects the nervous system. Drug Discov Today. 2013; 18: 818-35.

11. Maria C, Di G. The clinical spectrum of the neurological involvement in vasculitides. J Neurol Sci [Internet]. 2009; 285(1-2): 13-21. 